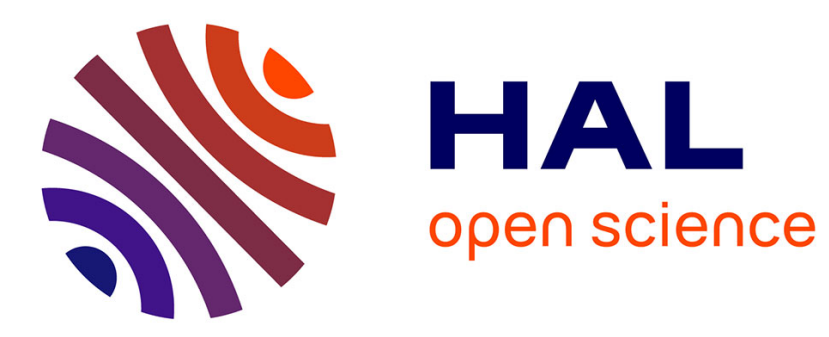

\title{
Terahertz Frequency Conversion with a Photomixer
}

Florin Lucian Constantin

\section{To cite this version:}

Florin Lucian Constantin. Terahertz Frequency Conversion with a Photomixer. Nonlinear Photonics, Jul 2020, Montréal, Canada. pp.1-2, 10.1364/NP.2020.NpTu4D.13 . hal-03056930

\section{HAL Id: hal-03056930 \\ https://hal.science/hal-03056930}

Submitted on 11 Dec 2020

HAL is a multi-disciplinary open access archive for the deposit and dissemination of scientific research documents, whether they are published or not. The documents may come from teaching and research institutions in France or abroad, or from public or private research centers.
L'archive ouverte pluridisciplinaire HAL, est destinée au dépôt et à la diffusion de documents scientifiques de niveau recherche, publiés ou non, émanant des établissements d'enseignement et de recherche français ou étrangers, des laboratoires publics ou privés.

$$
\text { Copyright }
$$




\title{
Terahertz Frequency Conversion with a Photomixer Florin Lucian Constantin \\ Laboratoire PhLAM, CNRS UMR 8523, 59655 Villeneuve d'Ascq, France FL.Constantin@univ-lille1.fr
}

\begin{abstract}
The nonlinear electrical response of a low temperature grown GaAs photomixer driven with the optical beat of two continuous-wave lasers is exploited in optical frequency downconversion for THz-wave modulation and heterodyne detection. (C) 2020 The Author(s)
\end{abstract}

\section{Introduction}

The terahertz-waves have a recognized potential for several applications including medical research, atmospheric and astrophysical observation, high-speed communications, chemical sensing and security screening [1]. The optical frequency down-conversion with a photoconductive antenna (photomixing) allowed continuous-wave (cw) THz generation [2]. The approach exploited the optical beat of two cw lasers emitting at frequencies $f_{1}$ and $f_{2}$ to drive an ultrafast photodetector with quadratic response to the optical fields. Upon application of a dc bias voltage, the photocurrent, oscillating at the difference-frequency $f_{T H z}=\left|f_{1}-f_{2}\right|$ in the terahertz domain, was coupled to an antenna to generate THz-waves. Compact, room-temperature operated, cost-effective and low-power consuming THz photonic systems with optical frequency control based on photomixers provided narrow spectral linewidth, wide frequency tunability, high dynamic range and high signal-to-noise ratio. This contribution exploits nonlinear photoconduction in low temperature grown GaAs (LTG-GaAs) for THz-wave modulation and detection by photomixing. Precisely, the optical frequency down-conversion is performed with an alternative electric field applied to the photomixer electrodes either by modulating the bias voltage or by an incoming $\mathrm{THz}$ radiation received by the antenna. The nonlinear electrical response of the LTG-GaAs allows frequency mixing in the photoconductor of the optical beat with the alternative electric field, enabling direct current modulation of the photomixing signal [3] or, alternatively, down-conversion to the microwave domain of an incoming THz-wave with a suitably tuned optical beat [4].

\section{Results}

The optical frequency down-conversion is performed with two spatially superposed coherent optical waves, detuned by an angular frequency $\omega$ and carrying the same optical power P, that enter at normal incidence in a LTG-GaAs photoconductor. The photomixing model developed in this contribution is based on uniform unidimensional carrier transport directed along an electric field oriented in parallel with the surface of the photoconductor. The continuity equations allow to derive the expression of the current density inside the photoconductor :

$$
j(t)=\frac{2 e \eta P}{\hbar \bar{\omega} A d}\left[\tau_{n}(E) v_{n}(E)\left(1+\frac{\omega \tau_{n}(E) \sin (\omega t)+\cos (\omega t)}{1+\left(\omega \tau_{n}(E)\right)^{2}}\right)+\tau_{p}(E) v_{p}(E)\left(1+\frac{\omega \tau_{p}(E) \sin (\omega t)+\cos (\omega t)}{1+\left(\omega \tau_{p}(E)\right)^{2}}\right)\right]
$$

where the laser beams, carrying nearly the same energy $\hbar \bar{\sigma}$, make an optical beat which is focused on a photoconductive area A, absorbed within a depth $d$, with a quantum efficiency $\eta$. The dependences of the carrier lifetimes $\tau_{\mathrm{n}, \mathrm{p}}(E)$ with the electric field [5] are linearized here, by taking into account only the carrier heating :

$$
\tau_{n, p}(E) \approx \tau_{0 n, 0 p}\left[1+3 \lambda e E /\left(2 k_{B} T\right)\right]
$$

in function of the Boltzmann constant $k_{B}$, the electron charge $e$, the room temperature $T=300 \mathrm{~K}$, the low-field carrier lifetimes $\tau_{0 n, 0 p}$ and the distance $\lambda$ between Coulomb barriers in LTG-GaAs. The carrier drift velocities have a nonlinear dependence on the electrical field $[6,7]$, that reads :

$$
v_{n, p}(E)=v_{s n, s p} /\left[1+v_{s n, s p} /\left(\mu_{0 n, 0 p} E\right)\right]
$$

where $v_{s n, s p}$ are the saturation velocities and $\mu_{0 n, 0 p}$ are the low-field mobilities for electrons and holes. The electric field in the photoconductor is expressed in the parallel-plate approximation as an alternative electric field (amplitude $E_{A C}=V_{A C} / r$, angular frequency $\omega_{A C}$, electrode spacing $\left.r\right)$ superposed to a bias electric field $\left(E_{D C}=V_{D C} / r\right)$ :

$$
E(t)=V_{D C} / r+\left(V_{A C} / r\right) \times \cos \left(\omega_{A C} t\right)
$$


The electrical nonlinearity arising from $v_{n, p}(E)$ and $\tau_{n, p}(E)$ dependences yields a current density with spectral components at angular frequencies $\omega \pm \mathrm{n} \omega_{A C}$ (n integer). The amplitudes and phases of these spectral components may be analytically calculated with eq. (1-4) for a given $E_{D C}$ using a power series expansion in terms of $E_{A C}$. The alternative electric field induced by an incoming THz-wave on the antenna electrodes, $E_{A C}=\overrightarrow{\mathrm{E}}_{T H z}^{i n c} \cdot \vec{l}_{\text {eff }} / r$ depends on the incident THz-electric field $\overrightarrow{\mathrm{E}}_{T H z}^{i n c}$ and the effective vector length $\vec{l}_{\text {eff }}$ of the antenna. THz frequency downconversion may be performed by heterodyning a THz-wave with the optical beat exploited as local oscillator (LO).

The experiment (Fig. 1.A) is based on a LTG-GaAs photomixer with a log-spiral antenna driven by the optical beat of two extended cavity laser diodes emitting at $820 \mathrm{~nm}$. The extremities of the antenna are connected to a microwave line addressed with a bias- $\mathrm{T}$, that allows to couple the modulation signal superposed to the bias voltage and to measure the photomixing signal on a microwave spectrum analyser. First, the modulation of the photomixing current is observed by coupling a radiofrequency signal to the photomixer operated with an optical beat in the microwave domain. The spectrum of the photomixing signal (Fig. 1.B) displays the carrier and the sidebands at $\omega \pm \omega_{A C}$ with nearly the same amplitude and other less intense sidebands. The amplitudes of the spectral components are calculated with eq. (1-4) using the modulation parameters, the LTG-GaAs photoconductor parameters from refs. [5-7], and the set of photomixer parameters. The results are multiplied with a common factor in order to match the amplitudes of the most intense spectral components and plotted with bars. The calculated and measured amplitudes agree for $\omega \pm 2 \omega_{A C}$ sidebands. The model may be improved by taking into account spatial dependence of the current density, optical power and spatial charge separation effects in the photoconductor. Alternatively, the photomixer is exploited for heterodyne detection of a THz-wave generated with a frequency multiplier driven by a microwave synthesizer (Fig. 1.C). A signal at $102.54 \mathrm{GHz}$, down-converted to $\sim 0.749 \mathrm{GHz}$, was recorded with a signal-to-noise ratio of 7.3 in $300 \mathrm{kHz}$ bandwidth. The responsivity is at the $10^{-3} \mathrm{~A} / \mathrm{W}$ level. The linewidth of the signal is related to the spectral purity of the diode lasers locked with the Pound-Drever-Hall technique to a Fabry-Perot cavity. The heterodyne detection signal is sensitive to the THz-wave amplitude and phase and, via antenna's geometry, to the spatial mode and polarization state. The photomixing approach enables integration of the optical beat generation and electrically-driven optoelectronic frequency conversion in a photonic circuit for THz-wave generation and detection.
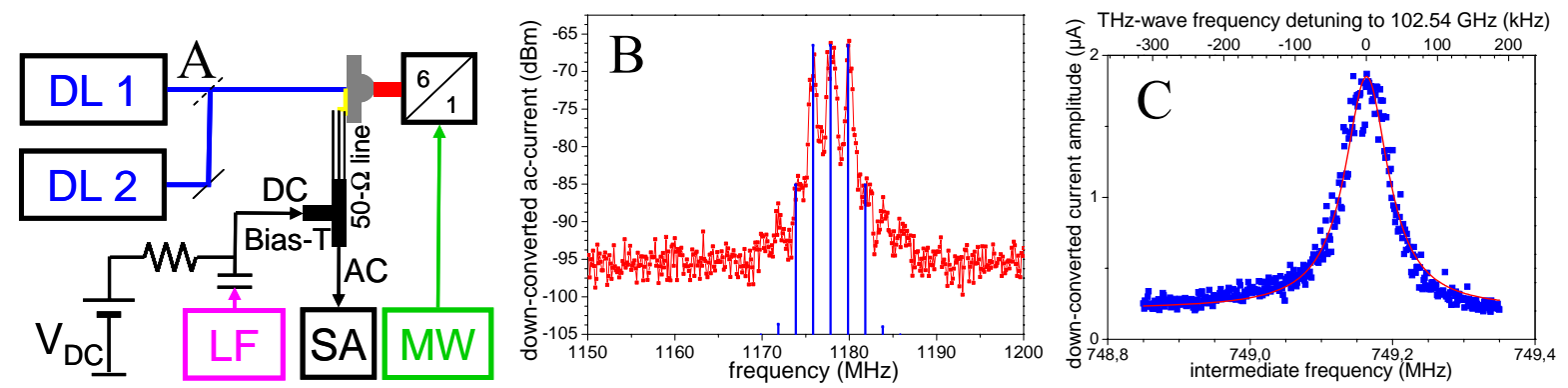

Fig. 1.A. The experimental setup. DL1, DL2 diode lasers, SA spectrum analyzer, LF radiofrequency synthesizer, MW microwave synthesizer. B. Spectrum of a modulated photomixing signal. Optical power $2 \times 20 \mathrm{~mW}$, optical beat at $\omega=2 \pi \times 1178 \mathrm{MHz}$. Modulation voltage $V_{A C}=0.5 \mathrm{~V}$ at $\omega_{A C}=2 \pi \times 2 \mathrm{MHz}$, bias voltage $\mathrm{V}_{D C}=0.517 \mathrm{~V}$. The calculated amplitudes of the spectral components are plotted with blue bars. Photomixer parameters used in the calculations: $\mathrm{r}=2 \mu \mathrm{m}, \mathrm{d}=2 \mu \mathrm{m}, \mathrm{A}=12.6 \mu \mathrm{m}^{2}$,

$\hbar \varpi / e=1.5 \mathrm{~V}, \eta=0.68$. RBW $=300 \mathrm{kHz}$, sweep time $4 \mathrm{~ms}, 2$ video averages. $\mathrm{C}$. Spectrum of the heterodyne detection signal of a

THz-wave at $\omega_{A C}=2 \pi \times 102.54 \mathrm{GHz}$. Bias voltage $V_{D C}=1 \mathrm{mV}$. Optical beat power $2 \times 14.5 \mathrm{~mW}$. RBW=300 kHz, sweep time 10 ms, 10 video averages. Lorentzian adjustment (red line) with a full-width at half-maximum linewidth of $74 \mathrm{kHz}$.

\section{References}

[1] S.S. Dhillon et al, "The 2017 terahertz science and technology roadmap," J. Phys. D 50, 043001 (2017).

[2] E.R. Brown et al, "Photomixing up to $3.8 \mathrm{THz}$ in low-temperature-grown GaAs," Appl. Phys. Lett. 66, $285-287$ (1995).

[3] F.L. Constantin, "Direct-current modulation of a photomixing signal," in Nonlinear Optics and its Applications IV, B.J. Eggleton, N.G.R. Broderick and A.L. Gaeta, eds., Proc. of SPIE Vol. 9894 (Society Of Photo-Optical Instrumentation Engineers, Bellingham, WA, 2016), pp. 98941K.

[4] F.L. Constantin, "Phase-coherent heterodyne detection in the terahertz regime with a photomixer," IEEE J. Quantum Electr. 47, 1458-1462 (2011).

[5] N. Zamdmer and Q. Hu, "Increase in response time of low-temperature-grown GaAs photoconductive switches at high voltage bias," Appl. Phys. Lett. 75, 2313-2315 (1999).

[6] E.R. Brown, “A photoconductive model for superior GaAs THz photomixer,” Appl. Phys. Lett. 75, 769-771 (1999).

[7] E.R. Brown et al, "Milliwatt output levels and superquadratic bias dependence in a low-temperature-grown GaAs photomixer," Appl. Phys. Lett. 64, 3311-3313 (1994). 